\title{
Conceptualization And Measurement Of University Technology Transfer Office Efficiency As A Formative Construct
}

\author{
Noorlizawati Abd Rahim, Zainai B. Mohamed, Astuty Amrin, Maslin Masrom ,Sya Azmeela \\ Shariff
}

\begin{abstract}
Although many universities have established Technology Transfer Office (TTO), there is no consensus in the literature that TTO has been successful in promoting academic entrepreneurship. Inhomogeneity of TTO services across different universities results in an inconsistent relationship between TTO formation and the rate of technology transfer activities. The TTO efficiency has been mostly researched based on the objective measures. However, far too little attention has been paid to explore the perception of academic entrepreneurs on the degree to which TTO has been efficient in greasing the wheels of their technology transfer pursuit. This paper aims to conceptualize TTO efficiency as a formative construct and to validate the proposed measurement model using partial least squares structural equation modeling (PLS-SEM). The empirical results confirmed the absence of collinearity while validity was established using a modified multitrait-multimethod (MTMM) matrix analysis. Furthermore, the indicators' weights revealed which constituent services provided by TTO benefit academic entrepreneurs the most. This study contributes new insights into academic entrepreneurs' perception towards the effectiveness of TTO services that further pave a way for the university to prioritize improvement measures.
\end{abstract}

Index Terms: Academic entrepreneurship, formative measurement model, modified multitrait-multimethod, partial Transfer Office.

\section{INTRODUCTION}

Technology Transfer Office (TTO) is one of the major area of interests within the field of academic entrepreneurship [1],[2]. The term TTO refers to an infrastructure established within a university to increase research discoveries commercialization [3] by managing technology transfer activities such as patenting, licensing and spin-offs creation [4],[5]. This semi-independent entity is responsible to identify research with commercial potential

Revised Manuscript Received on April 19, 2019.

Noorlizawati Abd Rahim, Dept. of Science, Management \& Design, Razak Faculty of Technology and Informatics, Universiti Teknologi

Zainai B. Mohamed, Dept. of Engineering, Razak Faculty of Technology and Informatics, Universiti Teknologi Malaysia, Kuala Lumpur, Malaysia.

Astuty Amrin, Dept. of Engineering, Razak Faculty of Technology and Informatics, Universiti Teknologi Malaysia, Kuala Lumpur, Malaysia.

Maslin Masrom, Dept. of Science, Management \& Design, Razak Faculty of Technology and Informatics, Universiti Teknologi Malaysia, Kuala Lumpur, Malaysia.

Sya Azmeela Shariff, Dept. of Advanced Informatics, Razak Faculty of Technology and Informatics, Universiti Teknologi Malaysia, Kuala Lumpur, Malaysia. least squares, structural equation modelling, Technology Malaysia, Kuala Lumpur, Malaysia.

[6] and to decide the best course of action to support its development towards successful technology transfer [7]. TTO is also regarded as a boundary spanner or a broker between academia and industry to assist scientists in understanding the needs of industry and to facilitate them in the technology transfer process [8].

Although many universities have established TTO, there is no consensus in the literature that it has been successful in promoting academic entrepreneurship. The inconsistent relationship between the TTO presence and the rate of technology transfer activities could be owing to the fact that TTO is not homogenous across different universities, particularly in terms of its relative emphasis on the type of supports offered to facilitate academic entrepreneurs. For instance, a scrutiny of 128 TTOs in American universities revealed TTO mission statements that prioritize more on licensing for royalties (78.7\%) and IP protection (75.2\%), than on facilitating academic entrepreneurs in the disclosure process $(71.6 \%)$ [9]. Hence, it remains unclear which constituent TTO services that are conducive and which inhibit academic entrepreneurs' technology transfer pursuits.

In accordance with these arguments, this paper attempts to conceptualize and validate TTO efficiency as a formative construct. The measure is intended to provide insights to higher education administrators on the improvement measures prioritization in promoting academic entrepreneurship.

\section{LITERATURE REVIEW}

\section{A. Technology Transfer Office}

Scholars have long debated the impact of TTO formation on the academic entrepreneurs' technology transfer pursuit [4],[10],[11],[12],[13]. For instance, a longitudinal study found that TTO establishment brings positive effect to the number of spin-offs generated by several public universities in Italy [10]. Similarly, another study by Hayter and Link [14] who analysed the data published by the Association of University Technology Managers, reported that the number of university start-ups have increased within the 15 years after the formation of a centre that provides services to improve research commercialization among universities in the United States.

Nonetheless, other researchers discovered contradict 
evidence that the presence of TTO plays a very limited role in increasing the technology transfer activities [11] and there is no direct linkage between the TTO establishment and the number of start-ups [12]. Indeed, the earlier positive findings have been strongly contested by some authors who criticize the increase in the number of start-ups could be a mere indicator that statistics have been improved since the TTO creation to capture the progress of technology transfer activities [15],[11]. Such inconsistent relationship implies that although there have been a diffusion of institutional initiative of TTO by many universities, there is no consensus in the literature that it has been successful in promoting academic entrepreneurship.

As a result, several studies have instead attempted to examine the relationship between the TTO capacity and the development of academic entrepreneurship. For instance, [16] drew the attention to think of the effect of TTO resources on the time it takes to commercialize technology, proposing a relationship between the numbers of TTO employee with the number of start-ups. Besides, there is research evidence showing a positive correlation between TTO size and the rate of start-ups creation [4].

Despite these positive findings, several studies however reached different conclusions. From the perspective of likelihood of scientists to create a start-up, [15] found that neither the TTO size nor its efficiency has influence on scientists' engagement in the technology transfer activities. Likewise, [11] who measured the TTO efficiency in terms of ratio of number of patent application to the number of TTO employees, unable to prove that greater commitment of TTO employees will results in higher propensity of entrepreneurially-oriented scientists to get involved in the start-ups creation.

Previous research regarding the TTO efficiency has been largely focused on the performance or productivity evaluation based on objective data rather than on perception of academic entrepreneurs. For instance, several studies attempted to assess the relative performance of TTO among universities in the United States [17-19] as well as in the United Kingdom [20]. Most of these studies measured the TTO productivity based on input-output indicators [21] and some evaluated efficiency using data envelopment analysis (DEA) approach [22]. These input indicators are such as annual invention disclosures, total number of patent applications, number of TTO employees, legal costs for IP protection and commercialization. On the other hand, the output indicators are such as the total number of patents granted, total number of start-ups created, annual number of licensing agreement and income.

Since the TTO efficiency has been mostly researched based on these objective measures, it would be more insightful to explore the perception of academic entrepreneurs on the degree to which TTO has been efficient in greasing the wheels of their technology transfer pursuit. By evaluating their perception on the efficiency of each different dimensions of services offered by TTO, this paper aims to provide opportunity to compare and prioritize services that need further improvements.

In Malaysia, TTO is quite a recent phenomenon which formally established in the early 21 st century, unlike those in the United States and the United Kingdom which have been in place since the 1980s [10]. A study comparing organization structures and roles of TTO between two research universities in Malaysia, highlighted that the TTO establishment is aimed to bridge the gap between academia and industry and as an alternative measure to traditional ineffective 'match-making' approach [7].

Several qualitative studies have identified TTO resources and capability among the factors that affect the technology transfer in some public research universities in Malaysia [23],[24]. Specifically, the interviews with several stakeholders including TTO managers and academic entrepreneurs provide insights on the need of competent and experienced TTO team to improve scientists' engagement in the technology transfer activities. Besides, [23] pointed out that nearly all of their interviewees agree on the role of TTO to provide collective services to smoothen the technology transfer process. The dimension of these services include managing intellectual property (IP) application, coordinating licensing, establishing spin-offs, marketing the technology as well as motivating scientists to participate in the technology transfer.

\section{B. Formative versus reflective measurement model}

Conventionally, the common measurement practice has been based upon a reflective measurement model, in which the indicators represent the effects or manifestations of an underlying latent construct. In a reflective measurement model, the direction of causality is from the construct to indicators implying that any changes in the construct will cause changes in all of the indicators simultaneously. Therefore, these indicators are interchangeable and should be highly correlated with each other. Besides, any indicators can generally be removed without changing the meaning of the construct provided that it has sufficient reliability [25].

Alternatively, a formative measurement model comprises indicators that cause or form a latent construct. From a statistical perspective, estimation of indicators' influence on the formative construct is based on multiple regression, thus justifying reason why the direction of causality is from the indicators to the construct. In contrast to reflective measurement model, indicators in formative measurement model are not interchangeable as they individually capture a specific aspect of a construct's domain [26]. Therefore, removal of any indicators should be carefully thought and justified as this potentially alters the meaning of the construct.

Most of the scales in management sciences [27] such as business and marketing researches [28] are based on reflective measurement model. However, [29] in their review of measurement model misspecification, claimed that $32 \%$ of the constructs in marketing literature have been measured incorrectly. They discovered that $31 \%$ of the measurements were modelled as reflective although they were more appropriately be modelled as formative. More importantly, they produced simulation results showing evidence that misspecification of even one-construct leads to errors and serious consequences in drawing theoretical 
conclusion from the entire model. Therefore, it is recommended for researcher to justify theoretically and empirically when designing a study whether the measurement of a particular construct should be modelled as reflective or formative [27],[29]. In this study, conceptualization and measurement of TTO efficiency as a formative construct are discussed in the methodology section.

\section{METHODOLOGY}

\section{A. Construct operationalization}

The construct TTO efficiency provides measure on the degree of TTO effectiveness to facilitate academic entrepreneurs' technology transfer pursuit. The TTO effectiveness is measured in terms of academic entrepreneurs' perception on various services efficiency offered by TTO as well as on the efficiency of TTO employees. A study by [3] offers preliminary insights derived from the literature on the type of key services, direct services and indirect services offered by TTO that may affect university's performance in the entrepreneurial activity. This collection of services include providing academic entrepreneurs the access to industry contacts, business partners, external investors as well as other resources from both public and private organizations. Based on an interview with TTO management at University A to confirm the type of services offered to scientists, this study introduced 4-items to measure academic entrepreneurs' perception on the supports received from TTO. As listed in Table I, the TTO effectiveness were measured in terms of efficiency of programs to increase understanding about commercialization, services to facilitate technology transfer (commercialization) activities, services to facilitate in finding business partners and services to facilitate in developing a financial plan. In addition, 1-item was included to measure academic entrepreneurs' perception on the efficiency of TTO employees in assisting them to commercialize research discoveries. Altogether, the respondents were asked to rate their level of agreement or disagreement on the 5-items according to a 6-point Likert scale ranging from (1) strongly disagree to (6) strongly agree.

Table I. Formative measurement indicators for assessing the effectiveness of Technology Transfer Office

\begin{tabular}{|c|c|c|}
\hline Indicator & Dimension & Descriptions \\
\hline TTO1 & Programs & $\begin{array}{l}\text { I think that programs } \\
\text { organized by TTO increase my } \\
\text { understanding about the } \\
\text { process of commercializing } \\
\text { research findings }\end{array}$ \\
\hline TTO2 & $\begin{array}{l}\text { Technology transfer } \\
\text { (commercialization) }\end{array}$ & $\begin{array}{l}\text { I think that the } \\
\text { * commercialization services } \\
\text { provided by TTO has assisted } \\
\text { me to commercialize research } \\
\text { findings ( } \\
\text { development, prototype } \\
\text { application, } \\
\text { technology } \\
\text { licensing/assignment). }\end{array}$ \\
\hline
\end{tabular}

I think that the services provided by TTO has assisted me to develop *financial plan to commercialize research findings ("business plan, market validation)

TTO4 Business partners I think that the *services provided by TTO has assisted me to find business partners to commercialize research findings ('marketing, promotion, exhibition)

TTO5 Staff efficiency I think that the TTO personnel are efficient in assisting me to commercialize research findings

\section{B. Justification for formative measurement model}

Five-decision criterions were justified to design the TTO efficiency as a formative construct. Based on the guidelines by [26], the first criterion was to justify the causality or the nature of relationship between construct and indicators. As listed in Table 1, indicators TTO1 (programs), TTO2 (services to commercialize), TTO3 (financial planning services), TTO4 (services to find partners) and TTO5 (efficiency of employees) define different aspects of TTO services domain. Therefore, the directions of causality in this case are from indicators to the construct. Considering the second criteria, the effectiveness of is not the traits that explain the indicators but rather combinations of indicators.

The third and fourth criterion were to justify whether the indicators represent consequences or causes of the construct and whether any changes in construct will cause changes in indicators. Programs, services to commercialize, financial planning services, services to find partners and efficiency of employees are causes that influence the overall effectiveness of TTO. This causing effect implies that any changes in these indicators will cause changes in the effectiveness of TTO construct. Moreover, low scores of effectiveness of TTO do not mean that a respondent perceives poor efficiency from all different dimensions of TTO1-TTO5. For instance, a respondent might perceive that TTO has been very efficient in assisting to develop financial plan (TTO3), but less efficient in other remaining dimensions (TTO1, TTO2, TTO4 and TTO5). Therefore, this explains that any changes in the construct will not necessarily cause changes in all of the indicators simultaneously.

Finally, the fifth decision criterion to design a formative construct was to justify whether the indicators are mutually interchangeable. Each indicators (TTO1-TTO5) are not having the same content and dropping any indicator will change the conceptual domain of the underlying construct. Therefore, it is expected that data analysis should show low correlations or an absence of collinearity between indicators of formative construct. Overall, the TTO efficiency construct was designed as a formative measurement model in this study since the five criterion suggested [26] have been justified. 


\section{Sample and data collection}

The sample consisted of academic entrepreneurs of one public research university in Malaysia (University A). The size of the target population is 1453 . Based on a power analysis [30],[26] using G*power software [31], the minimum sample size was determined as 92, with the maximum number of predictors set as five, effect size set as medium $(0.15)$ and power needed as 0.80 . The power was set as 0.80 following the guidelines by [32], which specify $80 \%$ as the minimum acceptable power across social science studies. Invitation mails to participate in the survey questionnaire were randomly emailed to academic scientists according to different type of engineering and technology departments. The maximum number of predictors was set as five since there were five dimensions of TTO efficiency being assessed. The list of all academic scientists was obtained from the official website of University A as well from the administrative office of the targeted departments. Only academic entrepreneurs or scientists who have been involved in the technology transfer pursuit were selected as valid respondents' sample. To ensure a valid response, respondents who scored 'never' to a questionnaire item that enquire if they have developed potential prototype, product, technology or process that can be commercialized by the industry were disregarded. Respondents must first qualify this item (at least answer 'very rarely involved') before their responses are valid to be considered. Finally, 115 valid questionnaires were received, exceeding the minimum sample size to proceed with data analysis.

\section{Data analysis technique}

Based on several guidelines [33],[26],[34],[25], following were the assessments performed to validate TTO efficiency as a formative construct:

\section{i. $\quad$ construct validity}

Construct validity assessment was performed to validate that the indicators of a construct are measuring what they are designed to. This study followed the procedures outlined by [35] to analyse a modified MultitraitMultimethod (MTMM) matrix for testing convergent validity of a formative construct [33, 34]. Discriminant validity was not assessed since only one construct was considered. Normalized values of all indicators (TTO1TTO5) and the weights were first obtained from partial least squares (PLS) estimations using SmartPLS 3.0 software. A composite scores labelled as TTO_CS were then calculated by summing up the indicators' weighted scores. These indicators' weighted scores were obtained by multiplying indicator's normalized value and its corresponding construct's PLS weight. Correlations between the interindicators and indicators-to-construct were then run using IBM SPSS Version 23 software to create the MTMM matrix. In general, convergent validity is attained if interindicators correlate highly for indicators that forming the same construct than with other indicators. Furthermore, the existence of significant indicators-to-construct correlations also demonstrates the convergent validity of a formative construct [35].

\section{ii. collinearity between indicators;}

Collinearity is a measure of high correlation between indicators that is desirable for reflective indicators but not for formative indicators. Substantial correlation between formative indicators (TTO1-TTO5) is undesirable because this implies that formative indicators are not sufficiently representing the distinctive aspects of the construct [34]. The presence of collinearity among formative indicators will affect the estimation of weights and statistical significance [26]. The magnitude of collinearity was assessed statistically based on tolerance and variance inflation factor (VIF). A VIF value higher than five indicates a potential presence of collinearity [26]. However, for a more rigorous test, [28],[36] proposed for VIF value to be less than 3.3 to indicate the absence of collinearity. In this study, VIF for all indicators were computed by following procedures to detect collinearity in IBM SPSS software outlined by [33].

\section{iii. $\quad$ significance and relevancy of indicators.}

The relative importance of each indicator was measured in terms of its outer weight, while the absolute importance of each indicator was measured in terms of its outer loading. Since the outer weights were the results of multiple regressions with the latent variable scores, each indicator's relative contribution to the construct can be determined by comparing the outer weights. It is necessary to assess the indicator's significance to confirm that the formative indicator truly contributes to the construct. The indicator is retained if its outer weight is significant or its outer loading is higher than 0.5 (for weight that is not significant) [26]. On the other hand, the indicator is removed if both the outer weight and outer loading are not significant. The outer weight significance testing was performed by computing the outer weights, outer loadings and t-values for TTO effectiveness construct using SmartPLS bootstrapping function.

\section{RESULTS}

The MTMM (Table II) shows that the indicator-toconstruct correlations for TTO1 (0.722), TTO2 (0.863), TTO3 (0.480), TTO4 (0.481), TTO5 (-0.358) were significant at the 0.01 level. TTO1-5 are the indicators' weighted scores. TTO_CS is the composite scores. These observations met one of the convergent validity criterion, which is the existence of significance correlations for indicators-to-construct [35]. In terms of correlations for inter-indicators, it was observed that all inter-indicators have significant correlations except for the TTO1- TTO3 pair (bolded values). The inter-indicator requirement by [35] were not fully met because of the theoretical nature of the formative measures that consist of indicators that are positively and negatively correlated [34]. As a result, the empirical data in this study provides partial evidence to show convergent validity of formative measurement model. Nonetheless, most studies involving formative measurement model disregard convergent validity in establishing validity procedures because of the lack of consensus concerning this 
assessment [34].

Table II. Inter-indicator and indicator-to-construct correlation matrix

\begin{tabular}{llllll}
\hline & TTO1 & TTO2 & TTO3 & TTO4 & TTO5 \\
\hline TTO1 & & & & & \\
TTO2 & $.510^{* *}$ & & & & \\
TTO3 & $\mathbf{0 . 0 4 9}$ & $.275^{* *}$ & & & \\
TTO4 & $.246^{* *}$ & $.514^{* *}$ & $.431^{* *}$ & & \\
TTO5 & $-.376^{* *}$ & $-.608^{* *}$ & $-.176^{*}$ & $-.555^{* *}$ & \\
TTO_CS & $.722^{* *}$ & $.863^{* *}$ & $.480^{* *}$ & $.481^{* *}$ & $-.358^{* *}$ \\
\hline
\end{tabular}

The collinearity statistics (Table III) show that the VIFs for all of the indicators were below 3.3, which met the condition to show the absence of collinearity [36]. Since the VIF check confirmed no critical levels of collinearity, the formative measurement model was adequately validated without the need to remove any indicators to proceed with interpretation of relative contribution of each formative indicators [26].

Table III. Collinearity statistics for TTO efficiency construct

\begin{tabular}{lll}
\hline & \multicolumn{2}{l}{ Collinearity Statistics } \\
\cline { 2 - 3 } Indicators & Tolerance & VIF \\
\hline TTO1 & 0.72 & 1.38 \\
TTO2 & 0.49 & 2.05 \\
TTO3 & 0.79 & 1.27 \\
TTO4 & 0.55 & 1.81 \\
TTO5 & 0.54 & 1.87 \\
\hline
\end{tabular}

The outer weight and loading significance testing results are shown in Table IV. Only one indicator did not have significant outer weight which was TTO4 (0.056). Although the corresponding outer loading was significant for TTO4, however the value was marginally below 0.5 . Since the value was only slightly below 0.5 , the indicator TTO4 can be considered to retain [26]. Furthermore, the deletion of any indicator is deemed necessary only if its outer loading is not significant and below 0.5 , which was not the case of TTO4.

The outer weights were the results of multiple regression between indicators and the construct with coefficient of determination (R2) value of 1.0. This implies that the indicators explain $100 \%$ of the construct and thus the outer weights can be compared to determine their relative contribution to the construct. The relative effect of each indicators was interpreted based on the guidelines by [37]. Significant indicators with positive weights (TTO2, TTO1 and TTO3) were compared based on their magnitudes. On the other hand, since the outer weights for TTO4 was not significant, its effects cannot be compared with the other indicators within the same construct. Significant indicator with negative weight like TTO5 (efficiency of TTO employees) was interpreted as having negative effect when the effects of other indicators within the same construct are controlled. Therefore, the results showed the relative effect of indicators towards TTO construct is given by commercialization services $(\mathrm{TTO} 2=0.730)$ as the strongest, followed by programs (TTO1=0.449) and services to develop financial plan $(\mathrm{TTO} 3=0.292)$.

Table IV. Outer weight and loading significance testing results

\begin{tabular}{cccc}
\hline Construct & Indicator & $\begin{array}{c}\text { Outer } \\
\text { Weights }\end{array}$ & $\begin{array}{c}\text { Outer } \\
\text { Loading }\end{array}$ \\
\hline & TTO1 & $0.449^{1}$ & $0.723^{1}$ \\
TTO & TTO2 & $0.730^{1}$ & $0.863^{1}$ \\
Effectiveness & TTO3 & $0.292^{2}$ & $0.480^{1}$ \\
& TTO4 & 0.056 & $0.481^{1}$ \\
& TTO5 & $-0.337^{2}$ & $0.358^{1}$ \\
\hline
\end{tabular}

\section{DISCUSSION, IMPLICATIONS AND LIMITATIONS}

The lack of research that evaluates TTO efficiency from academic entrepreneurs' perception motivated this study to conceptualize and measure TTO efficiency as a formative construct. This study performed construct validity, collinearity between indicators and significance assessments as suggested by previous scholars [33],[26],[34],[25] to validate the new five-item scale measuring TTO efficiency formatively. The outcome of this study provided an alternative perspective compared to the previous research, which commonly assess the effectiveness of TTO using objective measures.

In assessing the degree to which the establishment of TTO contributes to grease the wheels of academic entrepreneurs' technology transfer pursuit, the results showed that the service in patent application and technology licensing is the most important contributing role among the other services offered by TTO. The second most important contributing role of TTO is to organize programs to increase scientists' understanding about the technology transfer process. In accordance with [5], the findings also found a significant influence of TTO to facilitate scientists in developing financial planning as the third most important contributing role of TTO. Although [15] found no significant influence of staff efficiency, which was measured in terms of number of employees and the allocation of employees dedicated to patenting and licensing services, this does not appear to be the case in this study. Instead, it is intriguing to discover a significant inverse relationship between efficiency of TTO employees and overall effectiveness of TTO. These results infer that most of the respondents thought that the TTO employees have not been satisfactorily efficient in assisting their technology transfer pursuit. This observation could be partly explained by the fact that the establishment of TTO in University A is relatively new and still developing (TTO age: 7 years) in comparison to those in the developed countries (Average TTO age in the United States: 18.5 years, United Kingdom: 17.5 years, Spain: 18 years, Denmark: 13 years) [10].

Overall, these findings provide insights on the current

${ }^{1} p<0.01(\mathrm{t}>2.33)$

${ }^{2} p<0.05(\mathrm{t}>1.645)$ 
level of TTO efficiency at University A for the relevant authority to prioritize improvement measures by enhancing employees' efficiency (TTO5), by improving services to in developing a financial plan (TTO3). By modelling the construct formatively, the prioritization to implement improvement measures was easily recognized to focus on the dimensions with lower weight. Therefore, this study contributes to the academic entrepreneurship literature by revealing the importance of each dimension of TTO efficiency in the context of one public research university in Malaysia.

Nevertheless, several limitations exist and worth to be addressed in future studies. First, the characteristic of sample in this study was based on a single university investigation, thus limiting the generalization of findings to the other contexts. Second, the analysis was carried out by considering only the TTO efficiency as a contributing factor that facilitate academic entrepreneurs' technology transfer pursuit. Therefore, potential opportunity exists for further studies to cross-validate the proposed formative measurement model of TTO efficiency in a different context and to compare the influence of TTO efficiency with scientists' entrepreneurial characteristics or other cultural factors.

\section{REFERENCES}

1. Siegel, D.S. and M. Wright, Academic entrepreneurship: time for a rethink? British Journal of Management, 2015. 26(4): p. 582-595.

2. Grimaldi, R., et al., 30 years after Bayh-Dole: Reassessing academic entrepreneurship. Research Policy, 2011. 40(8): p. 1045-1057.

3. Rasmussen, E. and M. Wright, How can universities facilitate academic spin-offs? An entrepreneurial competency perspective. The Journal of Technology Transfer, 2015. 40(5): p. 782-799.

4. Muscio, A., D. Quaglione, and L. Ramaciotti, The effects of university rules on spinoff creation: The case of academia in Italy. Research Policy, 2016. 45(7): p. 13861396.

5. Slavtchev, V. and D. Göktepe-Hultén, Support for public research spin-offs by the parent organizations and the speed of commercialization. The Journal of Technology Transfer, 2016. 41(6): p. 1507-1525.

6. Gubitta, P., A. Tognazzo, and F. Destro, Signaling in academic ventures: the role of technology transfer offices and university funds. The journal of technology transfer, 2016. 41(2): p. 368-393.

7. Rahman, A., et al., Innovation \& Commercialization: Role of Technology Transfer Office in Malaysia Universities. Kamariah and Ahmad Anuar, Melati, Innovation \& Commercialization: Role of Technology Transfer Office in Malaysia Universities (November 9, 2011), 2011.

8. O'kane, C., et al., University technology transfer offices: The search for identity to build legitimacy. Research Policy, 2015. 44(2): p. 421-437.

9. Markman, G.D., et al., Entrepreneurship and university2005. 20(2): p. 241-263.

10. Rizzo, U. and L. Ramaciotti, The determinants of academic patenting by Italian universities. Technology Analysis \& Strategic Management, 2014. 26(4): p. 469483. facilitate scientists in acquiring partners (TTO4) as well as based technology transfer. Journal of business venturing,

11. Clarysse, B., V. Tartari, and A. Salter, The impact of entrepreneurial capacity, experience and organizational support on academic entrepreneurship. Research policy, 2011. 40(8): p. 1084-1093.

12. Nosella, A. and R. Grimaldi, University-level mechanisms supporting the creation of new companies: an analysis of Italian academic spin-offs. Technology Analysis \& Strategic Management, 2009. 21(6): p. 679698.

13. Phan, P.H., D.S. Siegel, and M. Wright, Science parks and incubators: observations, synthesis and future research. Journal of business venturing, 2005. 20(2): p. 165-182.

14. Hayter, C.S. and A.N. Link, On the economic impact of university proof of concept centers. The Journal of Technology Transfer, 2015. 40(1): p. 178-183.

15. Aldridge, T.T. and D. Audretsch, The Bayh-Dole act and scientist entrepreneurship. Research policy, 2011. 40(8): p. $1058-1067$.

16. Osiri, J., M.M. McCarty, and L. Jessup, Entrepreneurial culture in institutions of higher education: impact on academic entrepreneurship. Journal of Entrepreneurship Education, 2013. 16: p. 1.

17. Rogers, E.M., J. Yin, and J. Hoffmann, Assessing the effectiveness of technology transfer offices at US research universities. The Journal of the Association of University Technology Managers, 2000. 12(1): p. 47-80.

18. Tseng, A.A. and M. Raudensky, Performance evaluations of technology transfer offices of major US research universities. Journal of technology management \& innovation, 2014. 9(1): p. 93-102.

19. Kim, J., T. Anderson, and T. Daim, Assessing university technology transfer: A measure of efficiency patterns. International Journal of Innovation and Technology Management, 2008. 5(04): p. 495-526.

20. Chapple, W., et al., Assessing the relative performance of UK university technology transfer offices: parametric and non-parametric evidence. Research Policy, 2005. 34(3): p. 369-384.

21. Siegel, D.S., D. Waldman, and A. Link, Assessing the impact of organizational practices on the relative productivity of university technology transfer offices: an exploratory study. Research policy, 2003. 32(1): p. 27 48.

22. Thursby, J.G. and M.C. Thursby, Who is selling the ivory tower? Sources of growth in university licensing. Management science, 2002. 48(1): p. 90-104.

23. Khademi, T., et al., Enhancing commercialization level of academic research outputs in research university. Jurnal Teknologi, 2015. 74(4).

24. Othman, A., U. Haiyat, and A. Kohar, UniversityIndustry Technology Commercialization in Malaysia: Opportunities and Challenges. World Applied Sciences Journal (Innovation Challenges in Multidisciplinary Research \& Practice), 2014. 30: p. 167-184.

25. Lee, L., et al., On the use of partial least squares path modeling in accounting research. International Journal of Accounting Information Systems, 2011. 12(4): p. 305328.

26. Hair Jr, J.F., et al., A primer on partial least squares structural equation modeling (PLS-SEM). 2016: Sage publications.

27. Coltman, T., et al., Formative versus reflective measurement models: Two applications of formative measurement. Journal of Business Research, 2008 61(12): p. 1250-1262.

28. Diamantopoulos, A., P. Riefler, and K.P. Roth, 
Advancing formative measurement models. Journal of business research, 2008. 61(12): p. 1203-1218.

29. Jarvis, C.B., S.B. MacKenzie, and P.M. Podsakoff, A critical review of construct indicators and measurement model misspecification in marketing and consumer research. Journal of consumer research, 2003. 30(2): p. 199-218.

30. Cohen, J., Statistical power analysis for the behaviors science.(2nd). New Jersey: Laurence Erlbaum Associates, Publishers, Hillsdale, 1988.

31. Faul, F., et al., Statistical power analyses using $G^{*}$ Power 3.1: Tests for correlation and regression analyses. Behavior research methods, 2009. 41(4): p. 1149-1160.

32. Gefen, D., E.E. Rigdon, and D. Straub, Editor's comments: an update and extension to SEM guidelines for administrative and social science research. Mis Quarterly, 2011: p. iii-xiv.

33. Lowry, P.B. and J. Gaskin, Partial least squares (PLS) structural equation modeling (SEM) for building and testing behavioral causal theory: When to choose it and how to use it. IEEE transactions on professional communication, 2014. 57(2): p. 123-146.

34. Andreev, P., et al., Validating formative partial least squares (PLS) models: methodological review and empirical illustration. ICIS 2009 Proceedings, 2009: p. 193.

35. Loch, K.D., D.W. Straub, and S. Kamel, Diffusing the Internet in the Arab world: The role of social norms and technological culturation. IEEE transactions on engineering management, 2003. 50(1): p. 45-63.

36. Diamantopoulos, A. and J.A. Siguaw, Formative versus reflective indicators in organizational measure development: A comparison and empirical illustration. British Journal of Management, 2006. 17(4): p. 263-282.

37. Cenfetelli, R.T. and G. Bassellier, Interpretation of formative measurement in information systems research. MIS quarterly, 2009: p. 689-707. 